\title{
Schrifttum zum Studium des Strafrechts, Allgemeiner Teil
}

Der Bezug auf den GRUNDKURS STRAFRECHT, B.T., betrifft den GRUNDKURS STRAFrECHT, Die einzelnen Delikte, 5. Aufl. 1998.

\section{I. Älteres Schrifttum}

1. Lehrbücher und Grundrisse

BINDING

BINDING

BINDING

MAYER, M.E.

V. HIPPEL, ROBERT

BELING

v. LISZT/SCHMIDT

GERLAND

MEZGER

2. Kommentare

FRANK, $R$.

v. Olshausen
Handbuch des Strafrechts, Bd. I, 1885

Grundriß des Deutschen Strafrechts, Allgemeiner Teil, 8. Aufl. 1913, Nachdruck 1975

Die Normen und ihre Übertretung, Bd. I (4. Aufl. 1922), Bd. II (2. Aufl. 1914-1916), Bd. III (1918), Bd. IV (1919)

Der Allgemeine Teil des deutschen Strafrechts, 2. Aufl. 1923

Deutsches Strafrecht, Bd. I (1925), Bd. II (1930)

Grundzüge des Strafrechts, 11. Aufl. 1930

Lehrbuch des deutschen Strafrechts, Bd. I, Allgemeiner Teil, 26. Aufl. 1932

Deutsches Reichsstrafrecht, 2. Aufl. 1932

Strafrecht, Ein Lehrbuch, 3. (unv.) Aufl. 1949

Das Strafgesetzbuch für das Deutsche Reich, 18. Aufl. 1931 mit Nachtrag 1936 (Schäfer/v. Dohnanyi)

Kommentar zum Strafgesetzbuch für das Deutsche Reich, Bd. I, 12. Aufl. 1942/1943

\section{Neueres Schrifttum}

1. Einführung in das Strafrecht

HASSEMER

NAUCKE

ROXIN/ARZT/

TIEDEMANN

SCHMIDHÄUSER
Einführung in die Grundlagen des Strafrechts, 2. Aufl. 1990

Strafrecht, Eine Einführung, 8. Aufl. 1999

Einführung in das Strafrecht und Strafprozeßrecht,

3. Aufl. 1994

Einführung in das Strafrecht, 2. Aufl. 1984

2. Lehrbücher und Grundrisse

BAUMANN/WEBER/

MITSCH

BLEI

BOCKELMANN/VOLK

EBERT

ESER/BURKHARDT
Strafrecht, Allgemeiner Teil, 10. Aufl. 1995

Strafrecht I, Allgemeiner Teil, 18. Aufl. 1983

Strafrecht, Allgemeiner Teil, 4. Aufl. 1987

Strafrecht, Allgemeiner Teil, 2. Aufl. 1994

Strafrecht, Bd. 1: Allgemeine Verbrechenselemente, 4. Aufl. 1992 
ESER

FREUND

GROPP

HAFT

HAUF

HRUSCHKA

JAKOBS

JESCHECK/WEIGAND

KIENAPFEL

KÖHLER

KÜHL

MATT

MAURACH/ZIPF

MAURACH/GÖSSEL/ZIPF

MAYER, $\mathrm{H}$.

MAYER, $\mathrm{H}$.

ROXIN

SAUER

SCHLÜCHTER

SCHMIDHÄUSER

SCHMIDHÄUSER

STRATENWERTH

WELZEL

WESSELS/BEULKE

\section{Kommentare}

JOECKS

KOHLRAUSCH/LANGE

LACKNER/KÜHL

LEIPZIGER

KOMMENTAR (LK)

NOMOS KOMMENTAR (NK)

PFEIFFER/MAUL/SCHULTE

PREISENDANZ

SCHÖNKE/SCHRÖDER

SYSTEMATISCHER

KOMMENTAR (SK)

TRÖNDLE/FISCHER
STRAFRECHT Bd. 2: Fahrlässigkeit, Unterlassung, Versuch, Teilnahme, 3. Aufl. 1980

Strafrecht, Allgemeiner Teil, 1998

Strafrecht, Allgemeiner Teil, 1997

Strafrecht, Allgemeiner Teil, 8. Aufl. 1998

Strafrecht, Allgemeiner Teil, 1996

Strafrecht nach logisch-analytischer Methode, 2. Aufl. 1988

Strafrecht, Allgemeiner Teil, 2. Aufl. 1991

Lehrbuch des Strafrechts, Allgemeiner Teil, 5. Aufl. 1996

Strafrecht, Allgemeiner Teil, 5. Aufl. 1994

Strafrecht, Allgemeiner Teil, 1997

Strafrecht, Allgemeiner Teil, 2. Aufl. 1997

Strafrecht, Allgemeiner Teil, 1996

Strafrecht, Allgemeiner Teil, Tbd. 1, 8. Aufl. 1992

Strafrecht, Allgemeiner Teil, Tbd. 2, 7. Aufl. 1989

Das Deutsche Strafrecht, 2. Aufl. 1953

Strafrecht, Allgemeiner Teil, 1967

Strafrecht, Allgemeiner Teil, Bd. I, Grundlagen, Aufbau der Verbrechenslehre, 3. Aufl. 1997

Allgemeine Strafrechtslehre, 3. Aufl. 1955

Strafrecht, Allgemeiner Teil in aller Kürze, 3. Aufl. 2000

Strafrecht, Allgemeiner Teil, 2. Aufl. 1975

Strafrecht, Allgemeiner Teil, Studienbuch, 2. Aufl. 1984

Strafrecht, Allgemeiner Teil I, Die Straftat, 3. Aufl. 1981

Das deutsche Strafrecht, 11. Aufl. 1969

Strafrecht, Allgemeiner Teil, 29. Aufl. 1999

Studienkommentar StGB, 1999

Strafgesetzbuch mit Erläuterungen und Nebengesetzen, 43. Aufl. 1961

Strafgesetzbuch mit Erläuterungen, 23. Aufl. 1999

Großkommentar zum Strafgesetzbuch,

10. Aufl. $1978 \mathrm{ff}$, hrsg. v. Jescheck, Ruß und Willms (im Text als 10. Aufl. gekennzeichnet), 11. Aufl. $1992 \mathrm{ff}$, hrsg. v. Jähnke, Laufhütte und Odersky

zum Strafgesetzbuch, hrsg. v. Neumann und Schild, $1995 \mathrm{ff}$, Stand: Juni 1999

Strafgesetzbuch, Kommentar an Hand der Rechtsprechung des Bundesgerichtshofes, 1969

Strafgesetzbuch, Lehrkommentar, 30. Aufl. 1978

Strafgesetzbuch, bearb. von Cramer, Eser, Lenckner und Stree, 25. Aufl. 1997

zum Strafgesetzbuch, bearb. von Rudolphi, Horn, Hoyer, Günther, Samson, Allgemeiner Teil, 7. Aufl., Stand Sept. 1999 Strafgesetzbuch und Nebengesetze, 49. Aufl. 1999 
4. Kommentare zu strafrechtlichen Nebengesetzen

DALCKE/FUHRMANN/

SCHÄFER

ERBS/KOHLHAAS
Strafrecht und Strafverfahren, 37. Aufl. 1961

Strafrechtliche Nebengesetze (Loseblattsammlung)

\section{Strafrechtswissenschaftliche Zeitschriften}

Goltdammer's Archiv für Strafrecht (GA)

Monatsschrift für Kriminologie und Strafrechtsreform (MschrKrim)

Neue Zeitschrift für Strafrecht (NStZ)

Strafverteidiger (StV)

Zeitschrift für Wirtschaft, Steuer, Strafrecht (wistra)

Zeitschrift für die gesamte Strafrechtswissenschaft (ZStW)

\section{Verzeichnis der im Text angeführten Festschriften/Gedächtnisschriften}

BAUMANN, JÜRGEN

BENGL, KARL

BLAU, GÜNTER

BOCKELMANN, PAUL

BRUNS, HANS-JÜRGEN

BUNDESGERICHTSHOF

COING, Helmut

\section{DEUTSCHER}

JURISTENTAG

DREHER, EDUARD

DÜNNEBIER, HANNS

ENGISCH, KARL

GAGNÉR, STEN

GALLAS, WILHELM

GEERDS, FRIEDRICH

V. GLEISPACH, WENZEL

GOLTDAMMER's ARCHIV

GRÜNHUT, MAX

GRÜNWALD, GERALD

HANACK, ERNST WALTER

HEINITZ, ERNST

HENKEL, HEINRICH

HIRSCH, HANS JOACHIM

HONIG, RICHARD M.

JESCHECK, HANS-HEINR.

KAUFMANN, ARMIN

KAUFMANN, HILDE

KERN, EDUARD
Festschrift zum 70. Geburtstag, 1992, Bielefeld

Festschrift, 1984, München

Festschrift zum 70. Geburtstag, 1985, Berlin

Festschrift zum 70. Geburtstag, 1979, München

Festschrift zum 70. Geburtstag, 1978, Köln

Festschrift zum 25-jährigen Bestehen, „25 Jahre Bundesgerichtshof", 1975, München

Festschrift zum 70. Geburtstag, „Europäisches Rechtsdenken in Geschichte und Gegenwart", 1982, München

Festschrift zum 100-jährigen Bestehen, „Hundert Jahre

Deutsches Rechtsleben“, 1960, Karlsruhe

Festschrift zum 70. Geburtstag, 1977, Berlin

Festschrift zum 75. Geburtstag, 1982, Berlin

Festschrift zum 70. Geburtstag, 1969, Frankfurt

Festschrift zum 70. Geburtstag, 1991, München

Festschrift zum 70. Geburtstag, 1973, Berlin

Festschrift zum 70. Geburtstag, 1995, Lübeck

Festschrift zum 60. Geburtstag, „Gegenwartsfragen der Strafrechtswissenschaft", 1936, Berlin

Festschrift zum 140-jährigen Bestehen, 1993, Heidelberg

Erinnerungsgabe, 1965, Marburg

Festschrift zum 70. Geburtstag, 1999, Baden-Baden

Festschrift zum 70. Geburtstag, 1999, Berlin

Festschrift zum 70. Geburtstag, 1972, Berlin

Festschrift zum 70. Geburtstag, „Grundfragen der gesamten

Strafrechtswissenschaft", 1974, Berlin

Festschrift zum 70. Geburtstag 1999, Berlin

Festschrift zum 80. Geburtstag, 1970, Göttingen

Festschrift zum 70. Geburtstag, 1985, Berlin

Gedächtnisschrift, 1989, Köln

Gedächtnisschrift, 1986, Berlin

Tübinger Festschrift zum 80. Geburtstag, 1968, Tübingen 
KLEINKNECHT, THEODOR

KLUG, ULRICH

KOHLRAUSCH, EDUARD

KRAUSE, FRIEDRICH W.

LACKNER, KARL

LANGE, HEINRICH

LANGE, HERMANN

LANGE, RICHARD

LEFERENZ, HEINZ

LENCKNER THEODOR

MANGAKIS, GEORGIOS

ALEXANDROS

MARTENS, WOLFGANG

MAURACH, REINHART

MAYER, HELLMUTH

MESSNER, JOHANNES

MEYER, KARLHEINZ

MEZGER, EDMUND

MIYAZAWA, KOICHI

MOOS, REINHARD

NISHIHARA, HARUO

NOLL, PETER

OBERLANDESGERICHT

Celle

OEHLER, DIETRICH

PETERS, KARL

PFEIFFER, GERD

REBMANN, KURT

RITTLER, THEODOR

SALGER, HANNSKARL

SARSTEDT, WERNER

SCHAFFSTEIN, FRIEDR.

SCHMIDT, EBERHARD

SCHRÖDER, HORST

SCHÜLER-SPRINGORUM,

HORST

SCHWINGE, ERICh

V. SIMSON, WERNER
Festschrift zum 75. Geburtstag, „Strafverfahren im Rechtsstaat", 1985, München

Festschrift zum 70. Geburtstag, 1983, Köln

Festschrift zum 70. Geburtstag, „Probleme der Strafrechtserneuerung“, 1944, Berlin

Festschrift zum 70. Geburtstag, 1990, Köln

Festschrift zum 70. Geburtstag, 1987, Berlin

Festschrift zum 70. Geburtstag, 1990, München

Festschrift zum 70. Geburtstag, 1992, Stuttgart

Festschrift zum 70. Geburtstag, 1976, Berlin

Festschrift zum 70. Geburtstag, „Kriminologie-PsychiatrieStrafrecht", 1983, Heidelberg

Festschrift zum 70. Geburtstag, 1998, München

Festschrift, Strafrecht-Freiheit-Rechtsstaat, 1999, Athen

Gedächtnisschrift, 1987, Berlin

Festschrift zum 70. Geburtstag, 1972, Karlsruhe

Festschrift zum 70. Geburtstag, „Beiträge zur gesamten Strafrechtswissenschaft", 1966, Berlin

Festschrift zum 85. Geburtstag, „Ordnung im sozialen Wandel“", 1976, Berlin

Gedächtnisschrift, 1990, Berlin

Festschrift zum 70. Geburtstag, 1954, München

Festschrift, 1995, Baden-Baden

Festschrift zum 65. Geburtstag, 1997, Wien

Festschrift zum 70. Geburtstag, 1998, Baden-Baden

Gedächtnisschrift, 1984, Zürich

Göttinger Festschrift zum 250-jährigen Bestehen, 1961,

Göttingen

Festschrift zum 70. Geburtstag, 1985, Köln

Festschrift zum 70. Geburtstag, ,Einheit und Vielfalt des Strafrecht", 1974, Tübingen

Festschrift zum Abschied aus dem Amt als Präsident des Bundesgerichtshofes, „Strafrecht, Unternehmensrecht, Anwaltsrecht", 1988, Köln

Festschrift zum 65. Geburtstag, 1989, München

Festschrift zum 80. Geburtstag, 1957, Aalen

Festschrift zum Abschied aus dem Amt als Vizepräsident des BGH, 1995, Köln, München, u.a.

Festschrift zum 70. Geburtstag, 1981, Berlin

Festschrift zum 70. Geburtstag, 1975, Göttingen

Festschrift zum 70. Geburtstag, 1961, Göttingen

Gedächtnisschrift, 1978, München

Festschrift zum 65. Geburtstag, 1993, Köln, Berlin, Bonn, München

Festschrift zum 70. Geburtstag, „Persönlichkeit in der Demokratie“, 1973, Köln

Festschrift zum 75. Geburtstag, 1983, Baden-Baden 
SPANN, WolfGANG

SPENDEL, GÜNTER

STOCK, ULRICH

STREE, WALTER/

WESSELS, JOHANNES

TRIFFTERER, OTTO

TRÖNDLE, HERBERT

UNIVERSITÄT

HEIDELBERG

WALTER, ROBERT

WASSERMANN, RUDOLF

V. WEBER, HELMUTH

WEISSAUER, WALTHER

WELZEL, HANS

WOLF, ERNST

WOLFF, ERNST AMADEUS

WÜRTENBERGER, THOMAS

ZIPF, HEINZ
Festschrift zum 65. Geburtstag, „Medizin und Recht“, 1986, Berlin

Festschrift zum 70. Geburtstag, 1992, Berlin

Festschrift zum 70. Geburtstag, „Studien zur Strafrechtswissenschaft", 1966, Würzburg

Festschrift zum 70. Geburtstag, 1993, Heidelberg

Festschrift zum 65. Geburtstag, 1996, Wien u.a.

Festschrift zum 70. Geburtstag, 1989, Berlin

Festschrift der Juristischen Fakultät zur 600-Jahr-Feier,

„Richterliche Rechtsfortbildung“, 1986, Heidelberg

Festschrift zum 60. Geburtstag, 1991, Wien

Festschrift zum 60. Geburtstag, 1985, Darmstadt

Festschrift zum 70. Geburtstag, 1963, Bonn

Festschrift zum 65. Geburtstag, „Ärztliches Handeln - Verrechtlichung eines Berufsstandes“, 1986, Berlin

Festschrift zum 70. Geburtstag, 1974, Berlin

Festschrift zum 70. Geburtstag, „Recht und Rechtserkenntnis“, 1985, Köln

Festschrift zum 70. Geburtstag, 1998, Berlin u.a.

Festschrift zum 70. Geburtstag, „Kultur-Kriminalität-Strafrecht", 1977, Berlin

Gedächtnisschrift für Heinz Zipf, 1999, Heidelberg 
